This is the accepted manuscript version with post-publishing corrections of the following article: Comellas E., Bellomo F.J. and

Oller S., A generalized finite-strain damage model for quasi-incompressible hyperelasticity using hybrid formulation, International Journal for Numerical Methods in Engineering, 2015. doi:10.1002/nme.5118

INTERNATIONAL JOURNAL FOR NUMERICAL METHODS IN ENGINEERING

Int. J. Numer. Meth. Engng 2015; 00:1-23

Published online in Wiley InterScience (www.interscience.wiley.com). DOI: 10.1002/nme

\title{
A generalized finite strain damage model for quasi-incompressible hyperelasticity using hybrid formulation
}

\author{
Ester Comellas ${ }^{1,2 *}$, Facundo Bellomo ${ }^{3}$, Sergio Oller ${ }^{1,2}$ \\ ${ }^{1}$ Department of Strength of Materials and Structural Engineering, ETSECCPB, Universitat Politècnica de Catalunya, \\ Barcelona Tech (UPC), Campus Nord, Ed. C1, c/ Jordi Girona 1-3, 08034 Barcelona, Spain. \\ 2 International Center for Numerical Methods in Engineering (CIMNE), Campus Nord UPC, Ed. C1, c/ Gran Capità \\ $s / n, 08034$ Barcelona, Spain. \\ ${ }^{3}$ Facultad de Ingeniera, ICMASa, INIQUI (CONICET), National University of Salta (UNSA), Av. Bolivia 5150, 4400
} Salta, Argentina

\begin{abstract}
SUMMARY
A new generalized damage model for quasi-incompressible hyperelasticity in a total Lagrangian finite strain framework is presented. A Kachanov-like reduction factor $(1-D)$ is applied on the deviatoric part of the hyperelastic constitutive model. Linear and exponential softening are defined as damage evolution laws, both describable in terms of only two material parameters. The model is formulated following continuum damage mechanics theory such that it can be particularized for any hyperelastic model based on the volumetricisochoric split of the Helmholtz free energy. However, in the present work it has been implemented in an in-house finite element code for neo-Hooke and Ogden hyperelasticity. The details of the hybrid formulation used are also described. A couple of three-dimensional examples are presented to illustrate the main characteristics of the damage model. The results obtained reproduce a wide range of softening behaviors, highlighting the versatility of the formulation proposed. The damage formulation has been developed to be used in conjunction with mixing theory in order to model the behavior of fibered biological tissues. As an example, the markedly different behaviors of the fundamental components of the rectus sheath were reproduced using the damage model, obtaining excellent correlation with the experimental results from literature. Copyright (C) 2015 John Wiley \& Sons, Ltd.

Received ...
\end{abstract}

KEY WORDS: solids; constitutive equationsM damage; finite element methods; biomechanics

\section{INTRODUCTION}

Since Kachanov [1] first introduced the concept of effective stress through the use of a reduction factor associated with the amount of damage in a material, many authors have developed formulations based on this concept of elastic degradation to model damage or degradation in

${ }^{*}$ Correspondence to: Department of Strength of Materials and Structural Engineering, ETSECCPB, Universitat Politècnica de Catalunya, Barcelona Tech (UPC), Campus Nord, Ed. C1, c/ Jordi Girona 1-3, 08034 Barcelona, Spain. E-mail: ecomellas@cimne.upc.edu

Copyright (C) 2015 John Wiley \& Sons, Ltd.

Prepared using nmeauth.cls [Version: 2010/05/13 v3.00] 
materials. Over the years, these formulations have been consolidated and are now regarded as indisputable knowledge in the context of continuum damage mechanics [2, 3, 4, 5]. This phenomenological approach is based on a rigorous mathematical and thermodynamic basis and has proved to be a simple and effective tool in numerical modeling. Although initially formulated in an infinitesimal strain framework and as isotropic, it has been extended to include anisotropy (e.g. $[6,7])$, has been combined with plasticity (e.g. $[8,9,10])$ and viscoelasticity (e.g. [11]), and has been formulated for application to specific materials such as concrete (e.g. [12, 13]), composites (e.g. [14]) or biological tissues (e.g. [15]), among others.

The first damage models developed in a finite strain context were proposed more than two decades ago, being the work of Simo [16] one of the most renowned. These are generally based on the multiplicative decomposition of the deformation gradient into a volume-preserving or volumetric part and an isochoric or deviatoric part, with damage affecting only the latter. Like the formulations by Miehe [17], de Souza [18] and other authors [19], these models were motivated by the stress softening effect known as Mullins effect which is characteristic of rubber-like materials. More recently, damage models based on the decoupled volumetric-deviatoric response have been formulated to model the behavior of fibered soft biological tissues [20, 21].

All these formulations use damage criteria and evolution laws which are defined to particularly suit the specific material behavior being modeled. In this work, a new generalized softening finite strain model is proposed, which includes linear and exponential damage evolution laws that have
been translated from an infinitesirnal strain framevork [22] into the prescnt finite train one. The
novelty of this formulation is that, on the one hand, both proposed evolutions of the danage variable
are based on solely two measurable material properties and, on the other hand, the formulation can
be particularized for any decoupled volumetric-deviatoric hyperelastic constitutive model desired.
Thus, the result is a general-purtose formulation which is versatile enough to model disparate

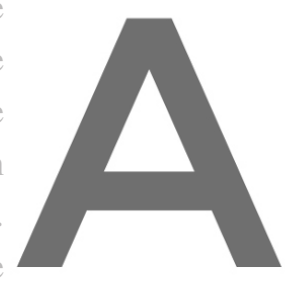

material behaviors without requiring reformulation of the damage model or complex material

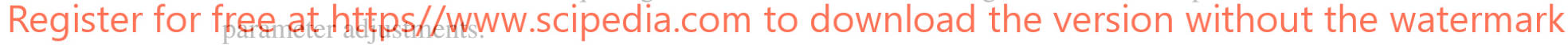

The ultimate aim, however, is to use this damage model in conjunction with mixing theory to represent the behavior of fibered soft biological tissues. The original theoretical framework of mixing theory was initially developed by Truesdell and Toupin [23]. It was later generalized, receiving the name of Series-Parallel Mixing Theory (S-P) [24], to take into account the incompatibility of deformations between its component materials and allowing, thus, the representation of complex behavior of composite materials, in this case a biological tissue, by means of the interaction between the simple constituent materials, each defined by its own constitutive law. In this way, the same damage model can be used to account for very different behaviors of the tissue components by particularizing the formulation to suit each component's needs.

The paper is organized as follows: Section 2 describes the new generalized damage formulation. This is then particularized for a neo-Hooke and an Ogden hyperelastic model and implemented in the in-house finite element code PLCd [25] using hybrid formulation, as described in Section 3. Examples for both particularizations are given in Section 4 with the aim of illustrating the main characteristics of the implemented formulation and the versatility of the model proposed. Finally, experimental data by Martins et al. [27] is numerically reproduced to show how the damage model proposed, in conjunction with mixing theory, allows for representing fibered soft biological tissues 
in which fiber and matrix have markedly different behaviors. The conclusions of the work are then stated in Section 5.

\section{GENERALIZED DAMAGE MODEL DEFINITION}

The multiplicative split of the deformation gradient typically used to represent the behavior of hyperelastic materials [28] results in a decoupled volumetric-isochoric Helmholtz free energy function, $\Psi$. Then, assuming that damage only affects the deviatoric part of the deformation [29], the Helmholtz free energy is of the form

$$
\Psi(\mathrm{C}, D)=\Psi_{\text {vol }}(J)+(1-D) \widetilde{\Psi}_{0}(\widetilde{\mathrm{C}})
$$

where $\widetilde{\Psi}_{0}$ is the undamaged isochoric or deviatoric part of the free energy and $\Psi_{v o l}$ is its undamaged volumetric part, both given in the reference configuration. The Jacobian determinant $J$ is related to the right Cauchy-Green strain tensor, $\mathbf{C}$, through $J=(\operatorname{det} \mathbf{C})^{1 / 2}$. The tilde in $\widetilde{\mathbf{C}}$ indicates that it is the deviatoric or volume-preserving part of $\mathbf{C}$, given by $\widetilde{\mathbf{C}}=J^{-2 / 3} \mathbf{C}$. The functions chosen for $\Psi_{\text {vol }}$ and $\widetilde{\Psi}_{0}$ must be such that $\Psi_{\text {vol }}(J)=0$ and $\widetilde{\Psi}_{0}(\widetilde{\mathbf{C}})=0$ hold if and only if $J=1$ and $\widetilde{\mathbf{C}}=\mathbf{I}$,
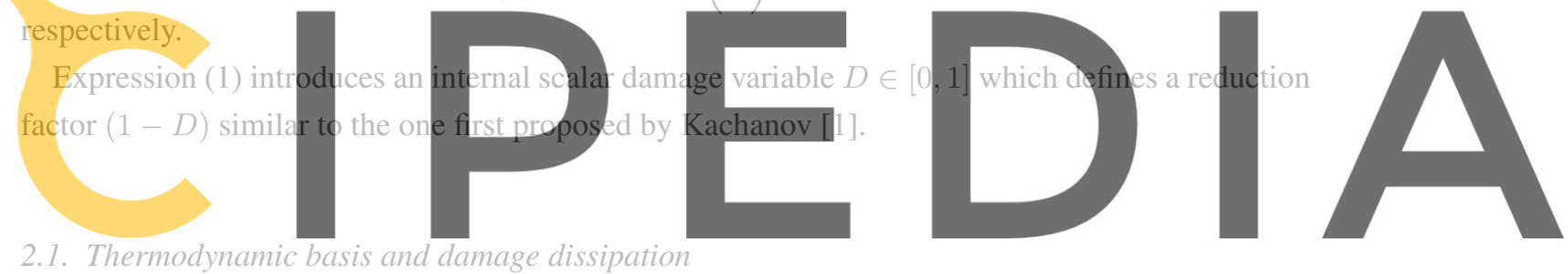

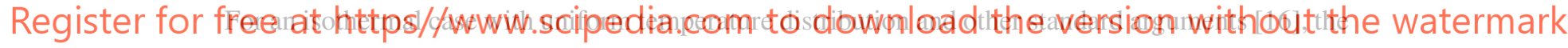

Clausius-Duhem inequality in the reference configuration is reduced to

$$
-\dot{\Psi}+\mathbf{S}: \frac{\dot{\mathbf{C}}}{2} \geq 0
$$

where $\mathbf{S}$ is the $2^{\text {nd }}$ Piola-Kirchhoff stress tensor. Considering $\Psi=\Psi(\mathbf{C}, D)$, the expression becomes

$$
-\left(\frac{\partial \Psi}{\partial D} \dot{D}+2 \frac{\partial \Psi}{\partial \mathbf{C}}: \frac{\dot{\mathbf{C}}}{2}\right)+\mathbf{S}: \frac{\dot{\mathbf{C}}}{2} \geq 0
$$

Then, introducing the free energy defined in (1) and rearranging, the internal dissipation in the reference configuration, $\Xi$, is obtained

$$
\Xi=\widetilde{\Psi}_{0} \dot{D}+\left[\mathbf{S}-2\left(\frac{\partial \Psi_{v o l}}{\partial \mathbf{C}}+(1-D) \frac{\partial \widetilde{\Psi}_{0}}{\partial \mathbf{C}}\right)\right]: \frac{\dot{\mathbf{C}}}{2} \geq 0
$$

This inequality must hold true for any strain increment, therefore, the term in brackets must be null and the expression of the dissipation is reduced to

$$
\Xi=\widetilde{\Psi}_{0} \dot{D} \geq 0
$$

Copyright (C) 2015 John Wiley \& Sons, Ltd.

Int. J. Numer. Meth. Engng (2015)

Prepared using nmeauth.cls

DOI: $10.1002 / \mathrm{nme}$ 


\subsection{Constitutive equation}

Setting the term in brackets in (4) to zero yields the following finite strain version of the Kachanov effective stress concept.

$$
\mathbf{S}=\mathbf{S}_{v o l}+(1-D) \widetilde{\mathbf{S}}_{0} \quad \text { with } \quad \mathbf{S}_{v o l}=-p J \mathbf{C}^{-1} \quad \text { and } \quad \widetilde{\mathbf{S}}_{0}=2 \frac{\partial \widetilde{\Psi}_{0}}{\partial \mathbf{C}}
$$

Here, the hydrostatic pressure $p=-\partial \Psi_{v o l} / \partial J$ has been introduced and the relation $\partial J / \partial \mathbf{C}=$ $J \mathbf{C}^{-1} / 2$ is used.

\subsection{Evolution of the damage internal variable}

The evolution of the damage variable is given by

$$
\dot{D}=\dot{\mu} \frac{\partial \mathcal{F}}{\partial \tau}
$$

where $\dot{\mu}$ is a non-negative scalar named damage consistency parameter used to define the loading, unloading and reloading conditions through the Kuhn-Tucker conditions
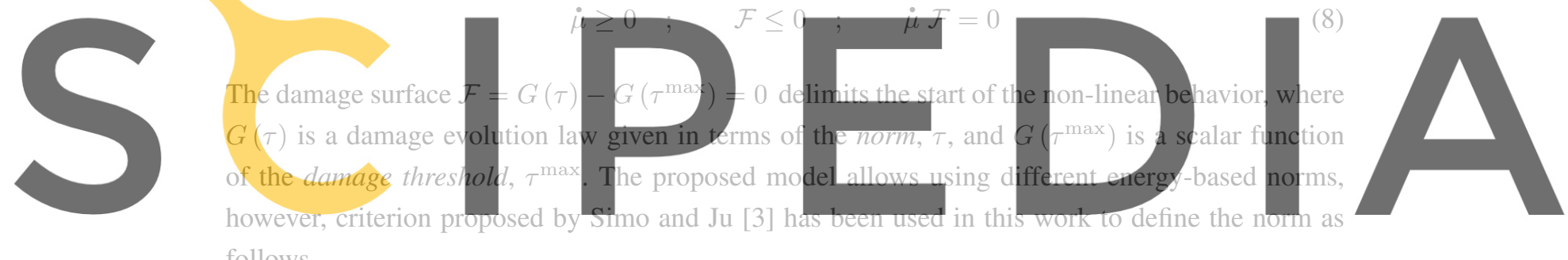

follows

Register for free at https//www.scipedia.com to download the version without(the watermark

The linear and exponential explicit scalar functions proposed in $[30,22]$ as damage evolution laws in an infinitesimal strain context have been translated to a finite strain framework to define $G(\tau)$. The advantage of these laws is that they are based on only two material parameters with direct physical sense that can be experimentally determined. Most evolution laws proposed up to date in finite strain damage formulations $[16,31,32,33,34]$ either require a considerable amount of parameters or rely on parameters that do not have a direct physical meaning, or both.

2.3.1. Linear softening The damage variable $D$ is defined as a scalar function with linear arguments

$$
D=G(\tau)=\frac{1-\frac{\tau_{0}^{d}}{\tau}}{1+H}
$$

where $\tau_{0}^{d}$ and $g_{f}^{d}$ are the material properties initial damage threshold and fracture energy per unit volume, respectively. $H$ is a parameter related to the dissipation obtained by imposing

$$
\int_{t_{0}}^{t_{\infty}} \Xi d t=g_{f}^{d}
$$


Introducing (5) and (10) into (11) yields

$$
\int_{t_{0}}^{t_{\infty}} \Xi d t=\int_{t_{0}}^{t_{\infty}} \widetilde{\Psi}_{0} \dot{D} d t=\int_{\tau_{0}}^{\tau_{\infty}} \widetilde{\Psi}_{0}(\tau) \frac{\partial G(\tau)}{\partial \tau} d \tau=\int_{G\left(\tau_{0}\right)}^{G\left(\tau_{\infty}\right)} \widetilde{\Psi}_{0}(\tau) d G(\tau)
$$

Considering the Simo and Ju criterion in (9), (12) can be further developed as follows by introducing integration by parts

$$
\int_{t_{0}}^{t_{\infty}} \Xi d t=\int_{G\left(\tau_{0}\right)}^{G\left(\tau_{\infty}\right)} \frac{1}{2} \tau^{2} d G(\tau)=\left.\frac{1}{2} \tau^{2} d G(\tau)\right|_{\tau_{0}} ^{\tau_{\infty}}-\int_{\tau_{0}}^{\tau_{\infty}} G(\tau) \tau d \tau
$$

The damage variable has been defined for the interval $D \in[0,1]$, therefore

$$
\left\{\begin{array}{l}
G\left(\tau_{0}\right)=0 \\
G\left(\tau_{\infty}\right)=1 \quad \Rightarrow \quad \frac{1-\frac{\tau_{0}^{d}}{\tau_{\infty}}}{1+H}=1 \quad \Rightarrow \quad \tau_{\infty}=-\frac{\tau_{0}^{d}}{H}
\end{array}\right.
$$

Then, (13) results in

$$
\int_{t_{0}}^{t_{\infty}} \Xi d t=-\frac{\left(\tau_{0}^{d}\right)^{2}}{2 H}
$$
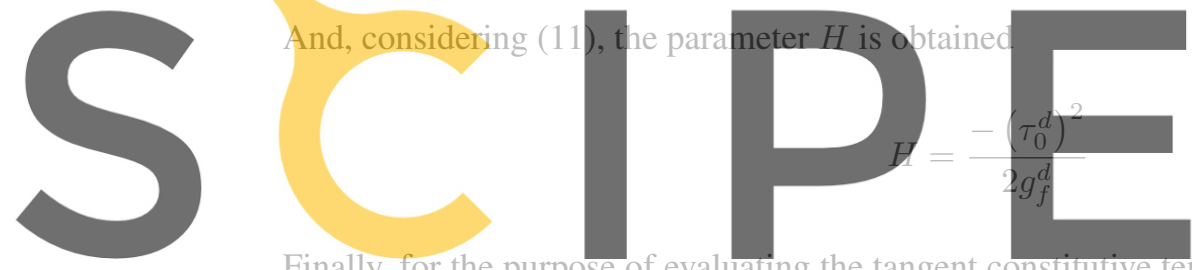

Finally, for the purpose of evaluating the tangent constitutive tensor defined tater in subsection 2.4 ,
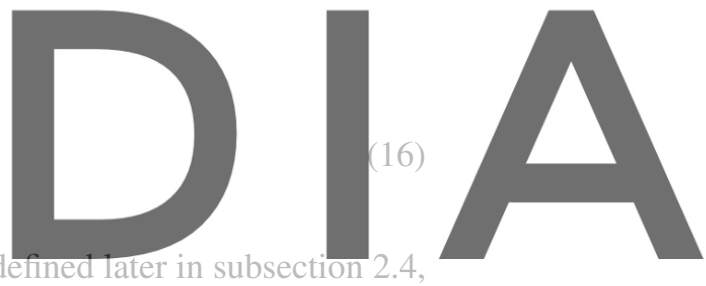

Register for free at https//www.scipedia.com to download the version without the watermark

$$
\frac{\partial G(\tau)}{\partial \tau}=\frac{-\tau_{0}^{d}}{\tau^{2}(1+H)}
$$

2.3.2. Exponential softening The damage variable $D$ is defined as a scalar function with exponential arguments

$$
D=G(\tau)=1-\frac{\tau_{0}^{d}}{\tau} \exp \left[A\left(1-\frac{\tau}{\tau_{0}^{d}}\right)\right]
$$

The parameter $A$ is obtained in a similar manner to the parameter $H$ in the linear softening law. Up to (13) the procedure is identical. Then, the damage variable, defined for the interval $D \in[0,1]$, is now

$$
\left\{\begin{array}{l}
G\left(\tau_{0}\right)=0 \\
G\left(\tau_{\infty}\right)=1 \quad \Longrightarrow \quad 1-\frac{\tau_{0}^{d}}{\tau_{\infty}} \exp \left[A\left(1-\frac{\tau_{\infty}}{\tau_{0}^{d}}\right)\right]=1
\end{array}\right.
$$

Since $\exp \left[A\left(1-\tau_{\infty} / \tau_{0}^{d}\right)\right]>0$ is always true, it becomes obvious that $\tau_{\infty} \rightarrow \infty$ for $G\left(\tau_{\infty}\right)=1$. Thus, operating on (13) with these values of $G(\tau)$ and $\tau$ yields

$$
\int_{t_{0}}^{t_{\infty}} \Xi d t=\left(\tau_{0}^{d}\right)^{2}\left(\frac{1}{A}+\frac{1}{2}\right)
$$


And, considering (11), the parameter $A$ is finally obtained

$$
A=\left[\frac{g_{f}^{d}}{\left(\tau_{0}^{d}\right)^{2}}-\frac{1}{2}\right]^{-1}
$$

The evaluation of the tangent constitutive tensor, performed in the following subsection, requires the differentiation of the evolution law with respect to the energy norm, which in this case is

$$
\frac{\partial G(\tau)}{\partial \tau}=\frac{\tau_{0}^{d}+A \tau}{\tau^{2}} \exp \left[A\left(1-\frac{\tau}{\tau_{0}^{d}}\right)\right]
$$

\subsection{Tangent constitutive tensor}

The material tangent constitutive tensor is known to be

$$
\mathbb{C}^{\tan }=4 \frac{\partial^{2} \Psi}{\partial \mathbf{C} \otimes \partial \mathbf{C}}
$$

Introducing the decoupled definition of the Helmholtz free energy in (1) and considering the definition in (6) with $D=G(\tau)$, the material elastic-damage tangent constitutive tensor obtained is
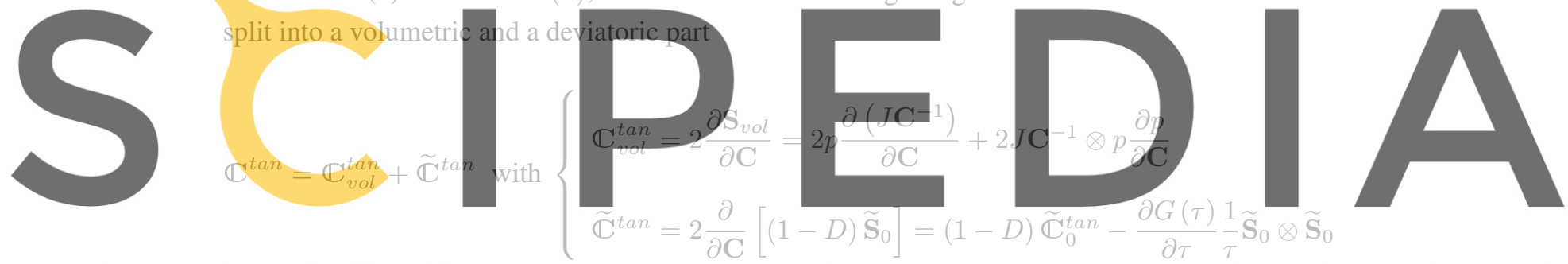

Register for free at https//www.scipedia.com to download the version without2the watermark where $\widetilde{\mathbb{C}}_{0}^{\tan }=2 \partial \widetilde{\mathrm{S}}_{0} / \partial \mathrm{C}$ is the deviatoric part of the undamaged hyperelastic model and $\partial G(\tau) / \partial \tau$ is defined in (17) and (22), for linear and exponential softening, respectively.

\section{NUMERICAL IMPLEMENTATION}

The proposed model has been integrated in the in-house finite element code PLCd [25], which is capable of solving large strain non-linear 3D solid mechanics problems. The code, developed in Fortran, uses a Pardiso solver [26] and a Full Newton algorithm to solve the problems. The model has been implemented in a total Lagrangian framework, particularizing the Helmholtz free energy in (1) for a neo-Hooke and an Ogden hyperelastic models. Any split quasi-incompressible hyperelastic model could be used (Yeoh, Mooney-Rivlin, Arruda-Boyce, Fung, etc.) but Neo-Hooke was chosen for its simplicity and exclusive dependence on physical parameters and Ogden was chosen for its capability of reproducing the stress-stretch J-curve characteristic of soft biological tissues. To overcome the numerical issues arising from incompressibility, a mixed $u / p$ formulation is used to solve the equilibrium equations. 


\subsection{Hybrid finite element formulation used}

The $u / p$ formulation implemented [35] is based on the classical displacement finite element method but includes an additional unknown variable, the pressure $p$, which is interpolated separately from the displacement variable $\mathbf{u}$. These elements are typically referred to as hybrid elements. The solid hexahedron with linear displacement and constant pressure (Q1P0) has been used in this work since it is the simplest functional available element of this type $[36,37]$. Then, the equations of motion for a typical element are

$$
\left[\begin{array}{cc}
\mathbf{K}_{u u} & \mathbf{K}_{u p} \\
\mathbf{K}_{p u} & \mathbf{K}_{p p}
\end{array}\right] \cdot\left[\begin{array}{c}
\mathbf{u} \\
p
\end{array}\right]=\left[\begin{array}{c}
\mathbb{F}^{e} \\
0
\end{array}\right]-\left[\begin{array}{l}
\mathbb{F}_{u}^{i n t} \\
\mathbb{F}_{p}^{i n t}
\end{array}\right]
$$

where $\mathbb{F}^{e}$ is the vector of nodal forces corresponding to the external loads and the $\mathbb{K}$ matrices and internal forces, $\mathbb{F}^{i n t}$, are given by

$$
\begin{gathered}
\mathbb{K}_{u u}=\int_{V_{0}} \mathbf{B}_{L}^{T}: \mathbb{C}^{\tan }: \mathbf{B}_{L} d V_{0}+\int_{V_{0}} \mathbf{B}_{N L}^{T} \cdot \mathbf{S} \cdot \mathbf{B}_{N L} d V_{0} \\
\mathbb{K}_{u p}=-\int_{V_{0}} \mathbf{B}_{N L}^{T} \cdot J \mathbf{C}^{-1} d V_{0}=\mathbb{K}_{p u}^{T}
\end{gathered}
$$

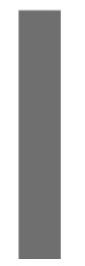

(26)

(27)

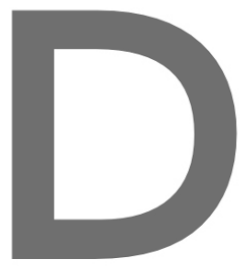

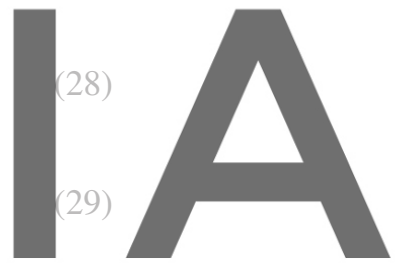

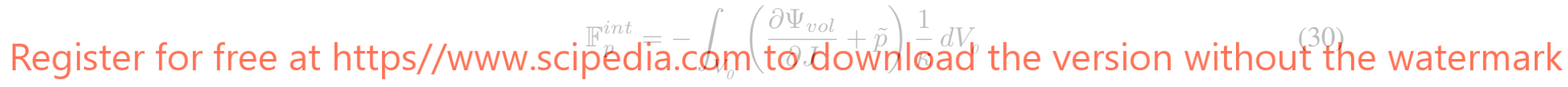

Here, $\mathbf{B}_{L}$ and $\mathbf{B}_{N L}$ are the classical linear and non-linear strain-displacement transformation tensors, respectively; $\tilde{p}$ is the pressure obtained by independent interpolation and $\kappa$ is the bulk modulus. Note that the bulk modulus, a material parameter of the constitutive model, works here as numerical penalizer.

Since there is a single pressure value per element, the equations of motion in (25) are condensed at elemental level and the equation to solve is reduced to

$$
\overline{\mathbf{K}} \cdot \mathbf{u}=\mathbb{F}^{e}-\overline{\mathbb{F}^{i n t}} \quad \text { with } \quad \overline{\mathbf{K}}=\left(\mathbf{K}_{u u}-\mathbf{K}_{u p} \mathbf{K}_{p p}^{-1} \mathbf{K}_{u p}^{T}\right) \quad \text { and } \quad \overline{\mathbb{F}^{i n t}}=\left(\mathbb{F}_{u}^{i n t}-\mathbf{K}_{u p} \mathbf{K}_{p p}^{-1} \mathbb{F}_{p}^{i n t}\right)
$$

The complete $u / p$ formulation algorithm implemented in PLCd is schematically described in Figure 1. 


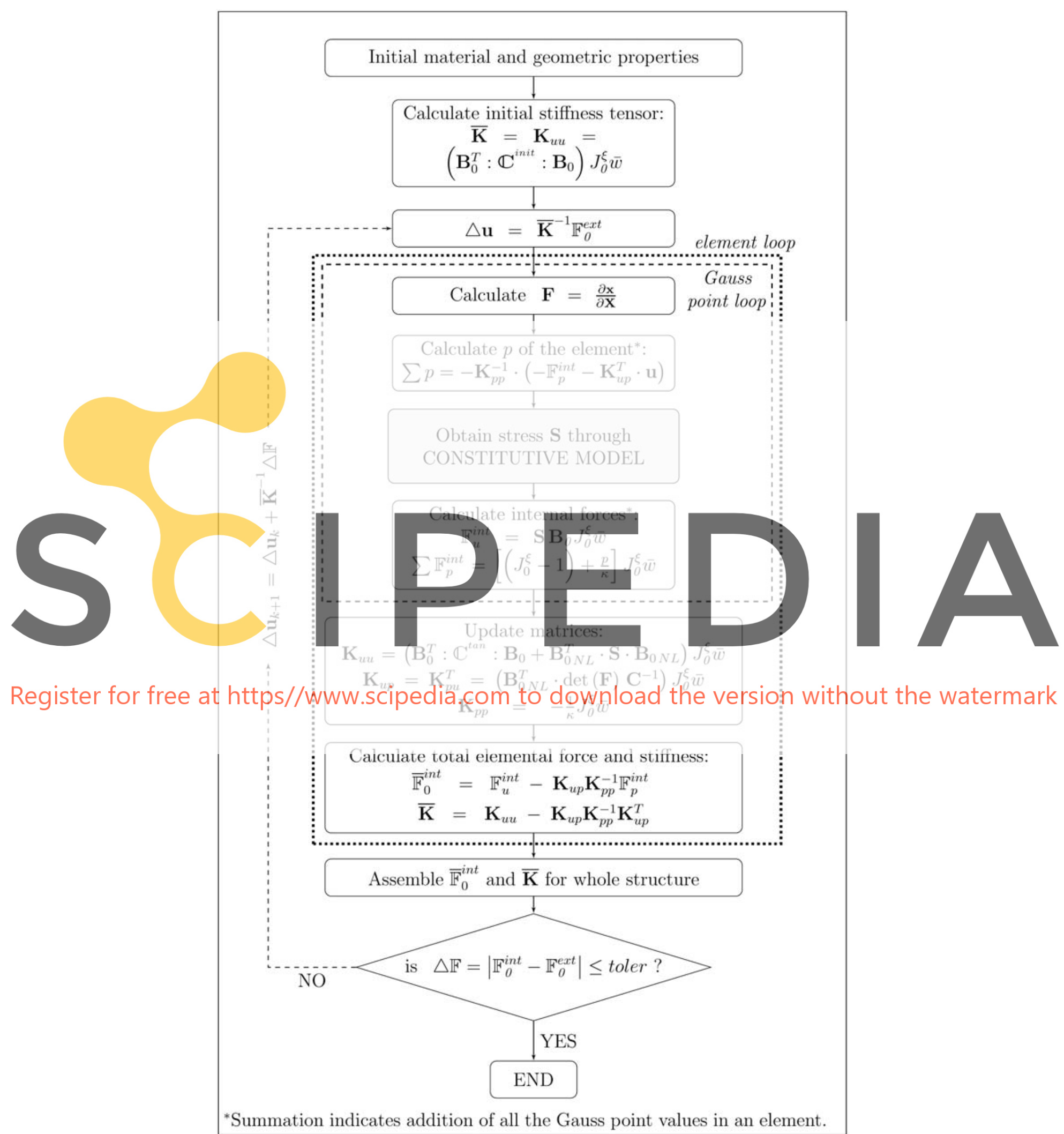

Figure 1 . Scheme of the $u / p$ or hybrid formulation implemented in the in-house code PLCd. 


\subsection{Model particularization for Neo-Hooken hyperelasticity}

The volumetric and deviatoric parts of the Helmholtz free energy in (1) particularized for an isotropic quasi-incompressible neo-Hookean model [36, 38] are:

$$
\left\{\begin{array}{l}
\Psi_{v o l}=\frac{\kappa}{2}(J-1)^{2} \\
\tilde{\Psi}=C_{1}\left(\tilde{I}_{\mathbf{C}}-3\right)
\end{array}\right.
$$

where $C_{1}$ is a material parameter given in terms of the shear modulus, $\mu$, as $C_{1}=\mu / 2$; and $\kappa$ is the bulk modulus. $\tilde{I}_{\mathbf{C}}=I_{\mathbf{C}}\left(I I I_{\mathbf{C}}\right)^{-1 / 3}$ is the modified volume-preserving first invariant of the right Cauchy-Green strain tensor $\mathbf{C}$. The first and third invariant of this tensor are $I_{\mathbf{C}}=\operatorname{tr}(\mathbf{C})=C_{i i}$ and $I I I_{\mathbf{C}}=\operatorname{det}(\mathbf{C})=J^{2}$, respectively. Through differentiation of these expressions, the complete expressions for the constitutive equation (6) and the tangent constitutive tensor (24) are obtained as

$$
\mathbf{S}=\mathbf{S}_{v o l}+(1-D) \widetilde{\mathbf{S}}_{0} \quad \text { with } \quad \mathbf{S}_{v o l}=-p J \mathbf{C}^{-1} \quad \text { and } \quad \widetilde{\mathbf{S}}_{0}=\mu J^{-2 / 3}\left(\mathbf{I}-\frac{1}{3} I_{\mathbf{C}} \mathbf{C}^{-1}\right)
$$
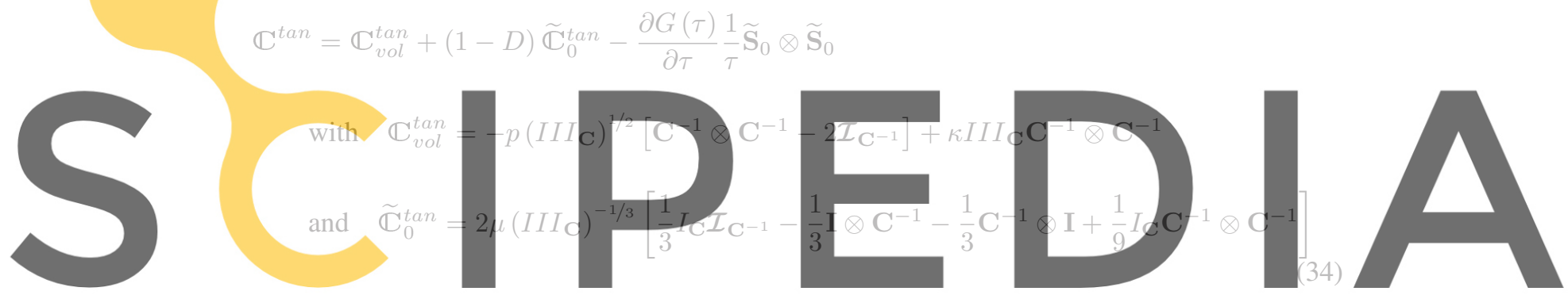

where the fourth-order tensor, $\mathcal{I}_{\mathrm{C}^{-1}}$, is

Register for free at https//www.scipedia.com to download the version without the watermark $\left[\mathcal{I}_{\mathrm{C}^{-1}}\right]_{I J K L}=\frac{1}{2}\left\{\left[\mathrm{C}^{-1}\right]_{I K}\left[\mathrm{C}^{-1}\right]_{J L}+\left[\mathrm{C}^{-1}\right]_{I L}\left[\mathrm{C}^{-1}\right]_{J K}\right\}$

\subsection{Model particularization for Ogden hyperelasticity}

The volumetric and deviatoric parts of the Helmholtz free energy in (1) particularized for an isotropic quasi-incompressible Ogden model $[28,39]$ are

$$
\left\{\begin{array}{l}
\Psi_{v o l}=\frac{\kappa}{2}(J-1)^{2} \\
\tilde{\Psi}=\sum_{i=1}^{3} \frac{\mu_{i}}{\alpha_{i}}\left(\tilde{\lambda}_{1}^{\alpha_{i}}+\tilde{\lambda}_{2}^{\alpha_{i}}+\tilde{\lambda}_{3}^{\alpha_{i}}-3\right)
\end{array}\right.
$$

where $\mu_{i}$ are (constant) shear moduli in the reference configuration and $\alpha_{i}$ are dimensionless constants, and both must satisfy the following consistency condition

$$
2 \mu=\sum_{i=1}^{3} \mu_{i} \alpha_{i} \quad \text { with } \quad \mu_{i} \alpha_{i}>0 \quad \text { for } \quad i=\{1,2,3\}
$$


Through derivation of these expressions, the complete expression for the constitutive equation (6) is obtained

$$
\mathbf{S}=\mathbf{S}_{v o l}+(1-D) \widetilde{\mathbf{S}}_{0} \quad \text { with } \quad \mathbf{S}_{v o l}=-p J \mathbf{C}^{-1} \quad \text { and } \quad \widetilde{\mathbf{S}}_{0}=\sum_{A=1}^{3} \beta_{A} \boldsymbol{M}_{A}
$$

where $\beta_{A}$ is related to the deviatoric principal stretches as follows

$$
\beta_{A}=\sum_{i=1}^{3} \mu_{i}\left(\tilde{\lambda}_{A}^{\alpha_{i}}-\frac{1}{3} \sum_{p=1}^{3} \tilde{\lambda}_{p}^{\alpha_{i}}\right)
$$

and the tensor $M_{A}$ is given by

$$
\boldsymbol{M}_{A}=\lambda_{A}^{-2} \boldsymbol{N}_{A} \otimes \boldsymbol{N}_{A}
$$

where $N_{A}$ is the eigenvector of the right Cauchy-Green deformation tensor: $\mathrm{C}=\sum_{A=1}^{3} \lambda_{A}^{2} N_{A} \otimes$ $N_{A}$.

Further derivation results in the complete expression for the tangent constitutive tensor in (24):

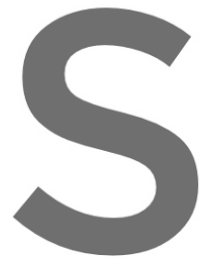

$$
\mathbb{C}^{\text {tan }}=\mathbb{C}_{\text {vol }}^{\text {tan }}+(1-D) \widetilde{\mathbb{C}}_{0}^{\tan }-\frac{\partial G(\tau)}{\partial \tau} \frac{1}{\tau} \widetilde{\mathbf{S}}_{0} \otimes \widetilde{\mathbf{S}}_{0}
$$
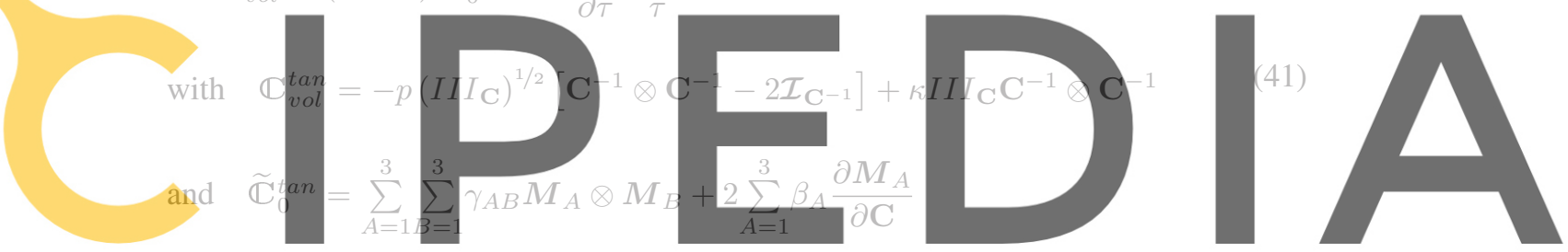

Here, the fourth-order tensor $\mathcal{I}_{\mathrm{C}^{-1}}$ is already defined in (35), $\gamma_{A B}$ is related to the deviatoric

Register for freque at hit

$$
\gamma_{A B}= \begin{cases}\sum_{i=1}^{3} \mu_{i} \alpha_{i}\left(\frac{1}{3} \tilde{\lambda}_{A}^{\alpha_{i}}+\frac{1}{9} \sum_{p=1}^{3} \tilde{\lambda}_{p}^{\alpha_{i}}\right) & \text { if } \quad A=B \\ \sum_{i=1}^{3} \mu_{i} \alpha_{i}\left(-\frac{1}{3} \tilde{\lambda}_{A}^{\alpha_{i}}-\frac{1}{3} \tilde{\lambda}_{B}^{\alpha_{i}}+\frac{1}{9} \sum_{p=1}^{3} \tilde{\lambda}_{p}^{\alpha_{i}}\right) & \text { if } \quad A \neq B\end{cases}
$$

and the derivative $\frac{\partial \boldsymbol{M}_{A}}{\partial \mathbf{C}}$ is given by

$$
\begin{aligned}
\frac{\partial \boldsymbol{M}_{A}}{\partial \mathbf{C}}= & \frac{1}{D_{A}}\left[i-\mathbf{I} \otimes \mathbf{I}+I I I_{\mathbf{C}} \lambda_{A}^{-2}\left(\mathbf{C}^{-1} \otimes \mathbf{C}^{-1}-\mathcal{I}_{\mathbf{C}^{-1}}\right)\right] \\
& +\frac{1}{D_{A}}\left[\lambda_{A}^{2}\left(\mathbf{I} \otimes \boldsymbol{M}_{A}+\boldsymbol{M}_{A} \otimes \mathbf{I}\right)-\frac{1}{2} \dot{D}_{A} \lambda_{A}\left(\boldsymbol{M}_{A} \otimes \boldsymbol{M}_{A}\right)\right] \\
& -\frac{1}{D_{A}}\left[I I I_{\mathbf{C}} \lambda_{A}^{-2}\left(\mathbf{C}^{-1} \otimes \boldsymbol{M}_{A}+\boldsymbol{M}_{A} \otimes \mathbf{C}^{-1}\right)\right]
\end{aligned}
$$


where the fourth-order identity tensor $i$ is

$$
i_{i j k l}=\frac{1}{2}\left(\delta_{i k} \delta_{j l}+\delta_{i l} \delta_{j k}\right)
$$

and the scalar $D_{A}$ and its derivative are

$$
\left\{\begin{array}{l}
D_{A}=2 \lambda_{A}^{4}-I_{\mathbf{C}} \lambda_{A}^{2}+I I I_{\mathbf{C}} \lambda_{A}^{2} \\
\dot{D}_{A}=8 \lambda_{A}^{3}-2 I_{\mathbf{C}} \lambda_{A}-2 I I I_{\mathbf{C}} \lambda_{A}^{-3}
\end{array}\right.
$$

The numerical integration in PLCd at Gauss point level of the generalized finite strain damage model presented in subsections 3.2 and 3.3 is outlined in Figure 2. The term $\left(\kappa I I I_{\mathbf{C}} \mathbf{C}^{-1} \otimes \mathbf{C}^{-1}\right)$ in the volumetric component of the tangent constitutive tensor of (34) and (41) is not included in the definition of the tangent tensor at constitutive level, since this term is already implicitly accounted for separately at element level in the implementation of the hybrid element schematized in Figure 1.

\section{NUMERICAL EXAMPLES}

The main characteristics of the generalized damage model proposed in the previous sections are presented here by means of two representative three-dimensional examples. A homogeneous state under uniaxial tension is reproduced with the aim of illustrating the basic constitutive characteristics of the damage formulation for both the neo-Hookean and the Ogden particularizations of the formulation.

Then, a membrane with a hole at its center is subjected to a tensile load in order to show how two different particularizations of the same formulation can result in very different damage initiation and evolution behaviors for a same specimen.

Finally, the experimental stress-stretch curve of a fibered soft biological tissue, the rectus sheath [27], is numerically reproduced. The damage formulation particularized for Ogden hyperelasticity is used to model the behavior of the tissue's constituents, whose overall response is obtained by means of the mixing theory. The aim is to show how this modeling approach allows accounting for considerably different damage softening behaviors of the constituents of a composite material such as fibered soft tissue.

\subsection{Homogeneous uniaxial tension}

An 8-noded hexahedral element with a single pressure point (Q1P0) is subjected to a displacementdriven pure tensile load state. Uniaxial tensile loading, unloading and reloading is imposed for both particularizations of the damage formulation to show how the choice of hyperelastic model has a direct influence on the response of the damage formulation. The stress-stretch response obtained for the neo-Hookean particularization is given in Figure 3 while Figure 4 shows the result obtained for the Ogden particularization. The linear damage evolution law given in (10) has been used in both cases, in addition to the specific material properties shown in the respective figures.

Both materials show a non-linear elastic response from the initial point $\mathrm{O}$ to point $\mathrm{A}$, where damage initiates. From A to B, loading continues but damage softening occurs. The gray dotted line 


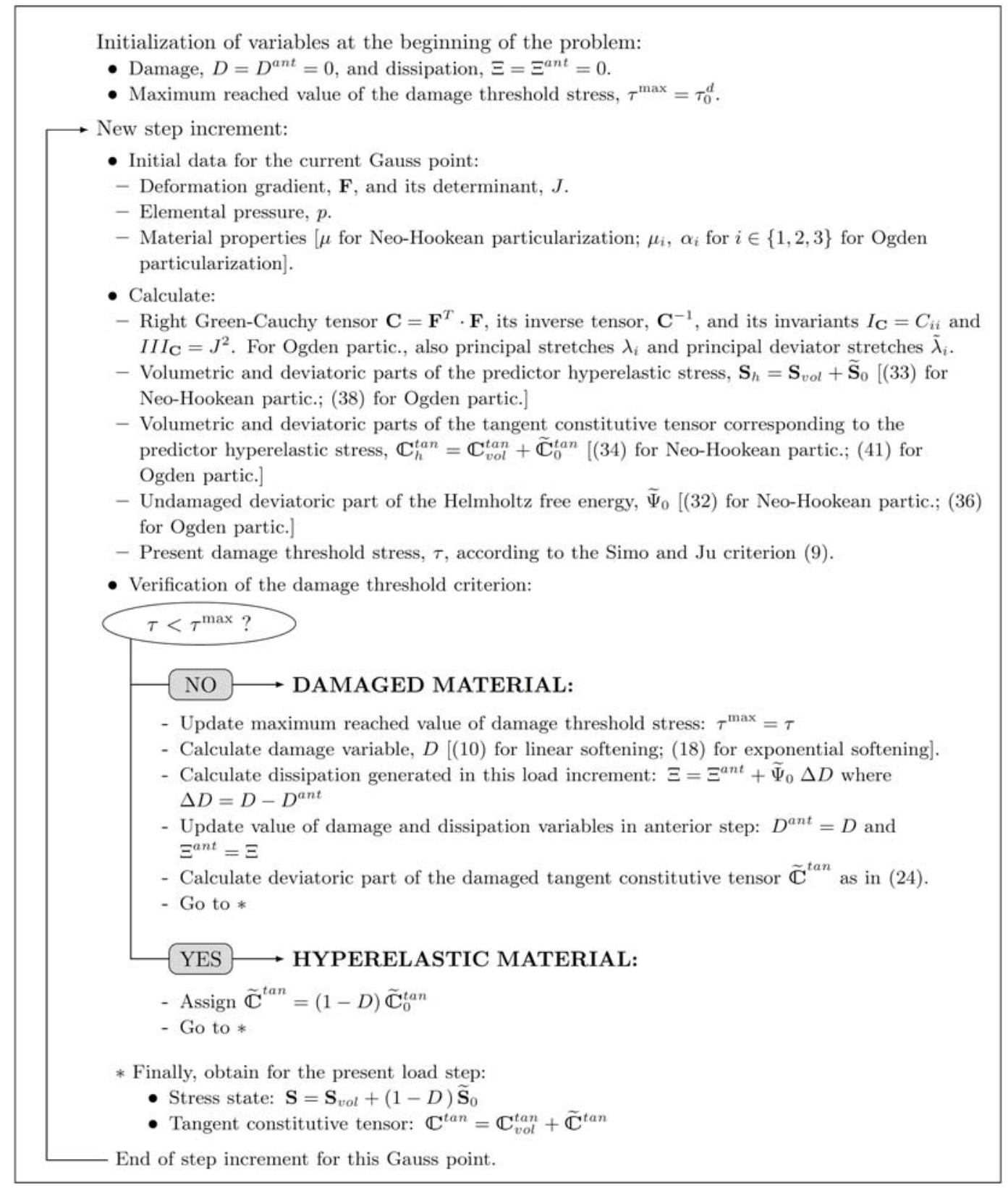

Figure 2. Numerical integration at a Gauss point of the finite strain damage model implemented in the inhouse code PLCd.

corresponds to the response of the undamaged (hyperelastic) material. In the neo-Hookean-based damage model, stress decreases as stretch increases once damage is initiated (point A), as opposed to the Ogden-based damage model, in which stress continues to grow with stretch, although with a much lower stiffness than the one of the undamaged model. At point B, unloading starts and stress decreases with the decreasing stretch, up to point $\mathrm{O}$, where loading is imposed again. The reloading path $(\mathrm{O}-\mathrm{B})$ is the same as the unloading one, with a stiffness lower than the original undamaged one (curve $\mathrm{O}-\mathrm{A}$ ). When reloading reaches the stretch value at which maximum damage had occurred previous to the unloading phase (point B), softening continues as if the unloading and reloading had 


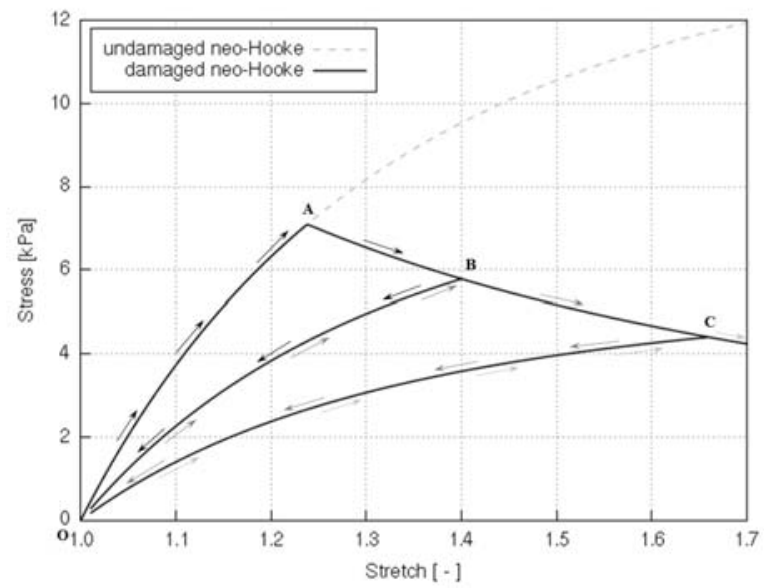

\begin{tabular}{ccc}
\hline $\begin{array}{c}\text { Material } \\
\text { parameter }\end{array}$ & Value & Units \\
\hline$C_{1}$ & 7.5 & $\mathrm{kPa}$ \\
$\kappa$ & 0.1 & $\mathrm{GPa}$ \\
$\tau_{0}^{d}$ & 57.7 & $\mathrm{~Pa}^{1 / 2}$ \\
$G_{f}$ & 20.0 & $\mathrm{kN} / \mathrm{m}$
\end{tabular}

Figure 3. $2^{\text {nd }}$ Piola-Kirchhoff stress vs. stretch for loading, unloading and reloading considering the linear damage evolution law with the neo-Hookean particularization of the damage formulation (left) and the material parameters used (right).

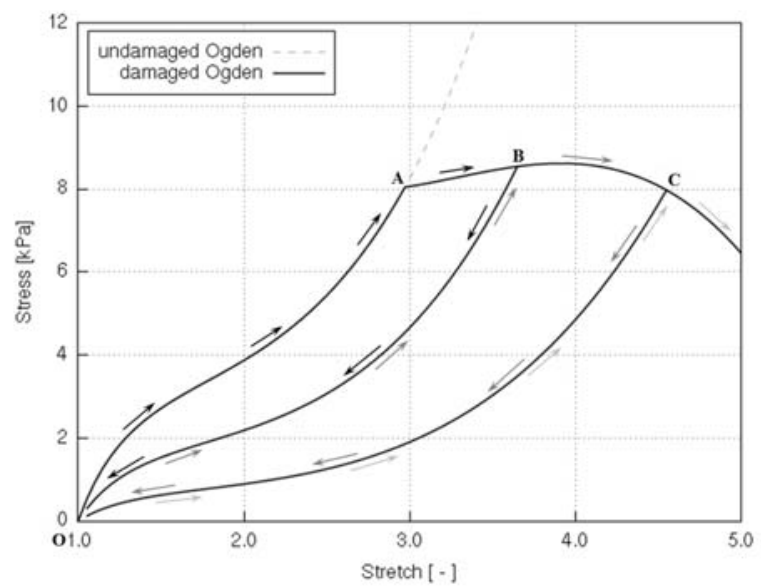

\begin{tabular}{ccc}
\hline $\begin{array}{c}\text { Material } \\
\text { parameter }\end{array}$ & Value & Units \\
\hline$\mu_{1}$ & 0.04 & $\mathrm{kPa}$ \\
$\mu_{2}$ & 3.7 & $\mathrm{kPa}$ \\
$\mu_{3}$ & -0.05 & $\mathrm{kPa}$ \\
$\alpha_{1}$ & 6.4 & - \\
$\alpha_{2}$ & 1.9 & - \\
$\alpha_{3}$ & -4.2 & - \\
$\kappa$ & 0.1 & $\mathrm{GPa}$ \\
$\tau_{0}^{d}$ & 2.31 & $\mathrm{kPa} 1 / 2$ \\
$G_{f}$ & 50.0 & $\mathrm{kN} / \mathrm{m}$ \\
\hline
\end{tabular}

Figure 4. $2^{\text {nd }}$ Piola-Kirchhoff stress vs. stretch for loading, unloading and reloading considering the linear damage evolution law with the Ogden particularization of the damage formulation (left) and the material parameters used (right).

not taken place. At point $\mathrm{C}$, unloading up to point $\mathrm{O}$ and reloading is imposed once more, exhibiting the same behavior as the first unloading-reloading phase (B-O-B).

As can be observed in these results, the damage model proposed is based on an accumulative discontinuous damage variable which can increase but never decrease, as imposed by the KuhnTucker conditions. This model is analogous to the infinitesimal strain model proposed by Oller [22], but translated into a finite strain framework in which large non-linearity is present, as made clear by the stress-stretch curves plotted in Figures 3 and 4 . Note that the generalized damage model can result in disparate softening behaviors, depending on the value of stiffness and amount of nonlinearity displayed by the original undamaged hyperelastic model chosen as basis for the generalized damage model. These dissimilarities are further enhanced depending on the combination of material 

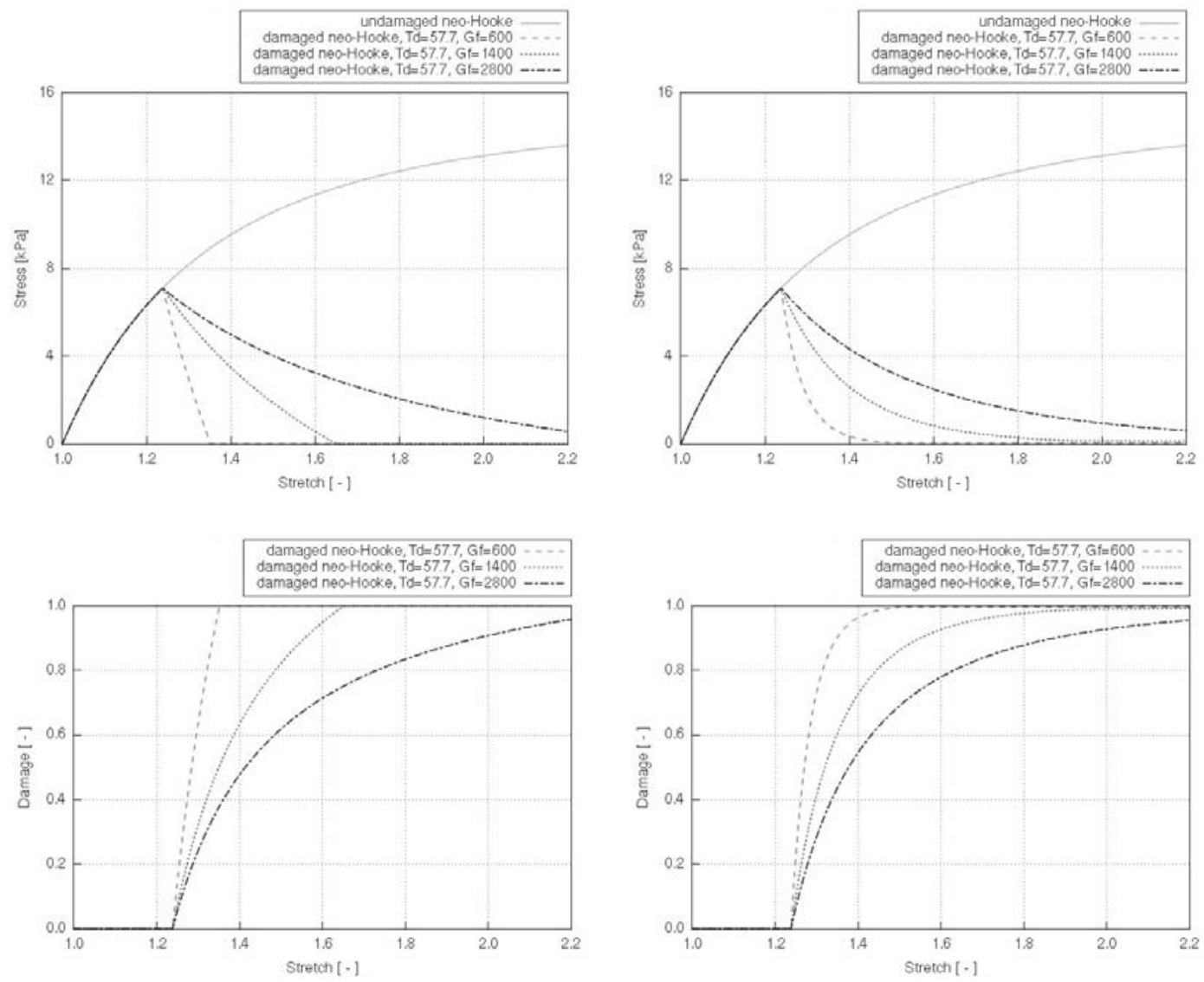

Figure 5. Results for the neo-Hookean particularization of the damage formulation for an initial damage threshold $\mathrm{Td}=57.7 \mathrm{~Pa}^{1 / 2}$ and different fracture energy values, Gf. $2^{\text {nd }}$ Piola-Kirchhoff stress vs. stretch considering the linear softening law (top left) and the corresponding evolution of the damage variable, $D$ (bottom left). $2^{\text {nd }}$ Piola-Kirchhoff stress vs. stretch considering the exponential softening law (top right) and the corresponding evolution of the damage variable, $D$ (bottom right).

parameter values used. The effect of changing the initial damage threshold $\tau_{0}^{d}$ and the maximum dissipated fracture energy $g_{f}^{d}$ values, as well as the type of damage evolution law selected, is illustrated in Figure 5 for the neo-Hookean particularization of the damage formulation and in Figure 6 for the Ogden one. In both figures, the gray solid line represents the undamaged (hyperelastic) response while the dotted lines show the response of the damage model for different combinations of material parameters, where Td is the initial damage threshold $\tau_{0}^{d}$ and Gf is the maximum dissipated fracture energy per unit of area, i.e., Gf $=g_{f}^{d} L_{0}$. Here, $L_{0}$ is the element's characteristic length in the reference configuration $[12,30]$. These figures show the stress-stretch curves obtained under uniaxial loading when using the linear and the exponential damage evolution laws and, below, the corresponding evolution of the internal damage variable, $D$.

It is interesting to observe how the use of the exponential damage evolution law in the neoHookean particularization of the model translates into a more markedly non-linear softening behavior in the stress-stretch response. Yet, the opposite effect is observed in some of the stressstretch responses of the Ogden particularization, for example the one obtained for $\mathrm{Td}=2.31 \mathrm{kPa}^{1 / 2}$ 

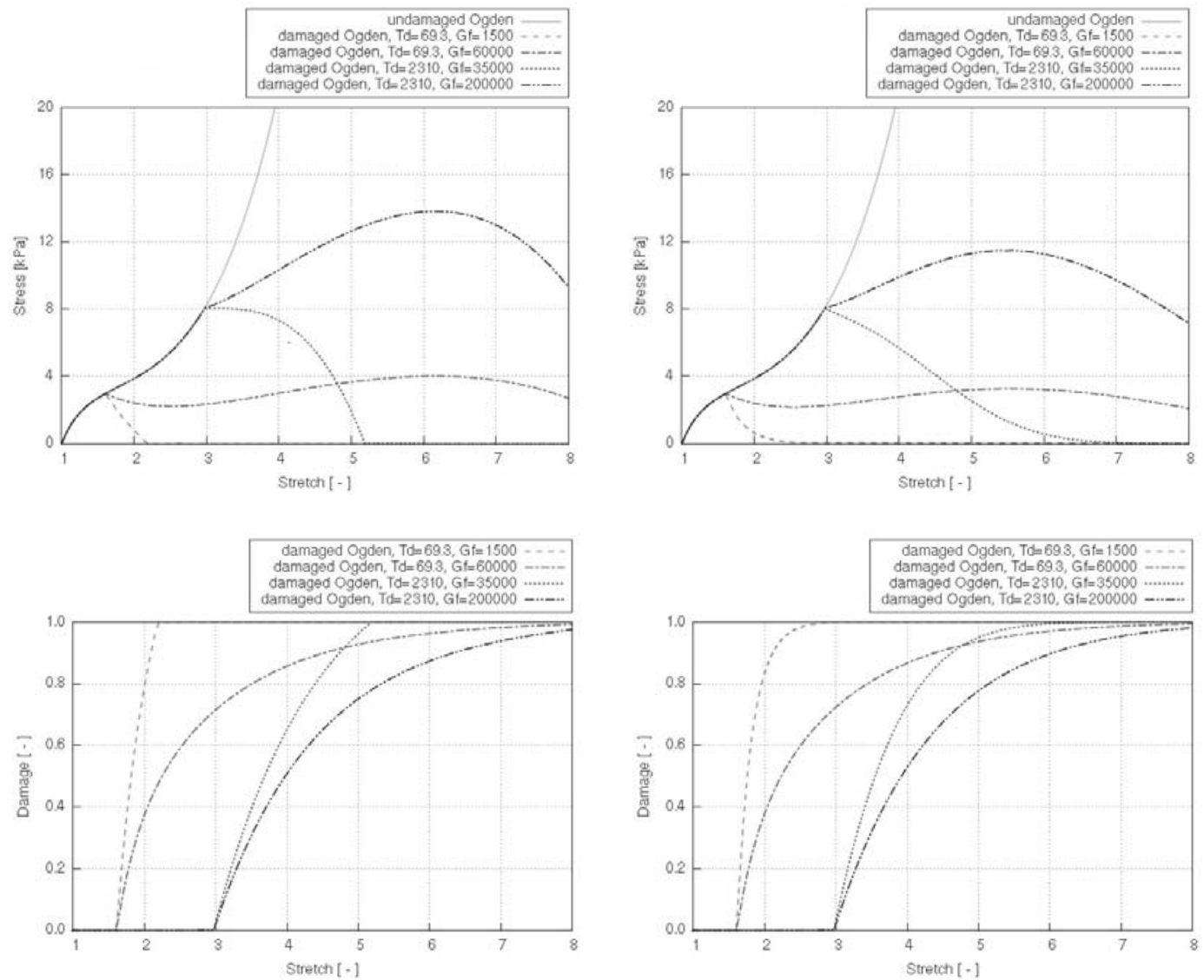

Figure 6. Results for the Ogden particularization of the damage formulation for different fracture energy, Gf, and initial damage threshold, Td, values. $2^{\text {nd }}$ Piola-Kirchhoff stress vs. stretch considering the linear softening law (top left) and the corresponding evolution of the damage variable, $D$ (bottom left). $2^{\text {nd }}$ PiolaKirchhoff stress vs. stretch considering the exponential softening law (top right) and the corresponding evolution of the damage variable, $D$ (bottom right).

and $\mathrm{Gf}=35 \mathrm{kN} / \mathrm{m}$. This is due to the interaction of the exponential softening with the highly nonlinear original undamaged (hyperelastic) curve.

\subsection{Membrane with a hole}

The membrane with a hole at its center depicted in Figure 7 is subjected to the indicated displacement-driven displacements $u$. Due to the symmetry in the specimen, only a quarter of the membrane has been discretized using 360 8-noded hexahedral elements with a single pressure point (Q1P0). Symmetry conditions are imposed, thus, nodes belonging to the symmetry $y-z$ plane shown in Figure 7 have motion restricted in the $x$-direction, while nodes belonging to the symmetry $x-z$ plane have motion in the $y$-direction restricted. A total of 500 accumulative incremental displacements are imposed in the $y$-direction on the nodes of the top part of the specimen, with the other directions left unrestrained.

The example is run for both the neo-Hookean and Ogden particularizations of the damage formulation. In the former, the material properties used are those defined in Figure 3, except for the fracture energy which is set to Gf $=600 \mathrm{kN} / \mathrm{m}$; while the latter uses the material properties 

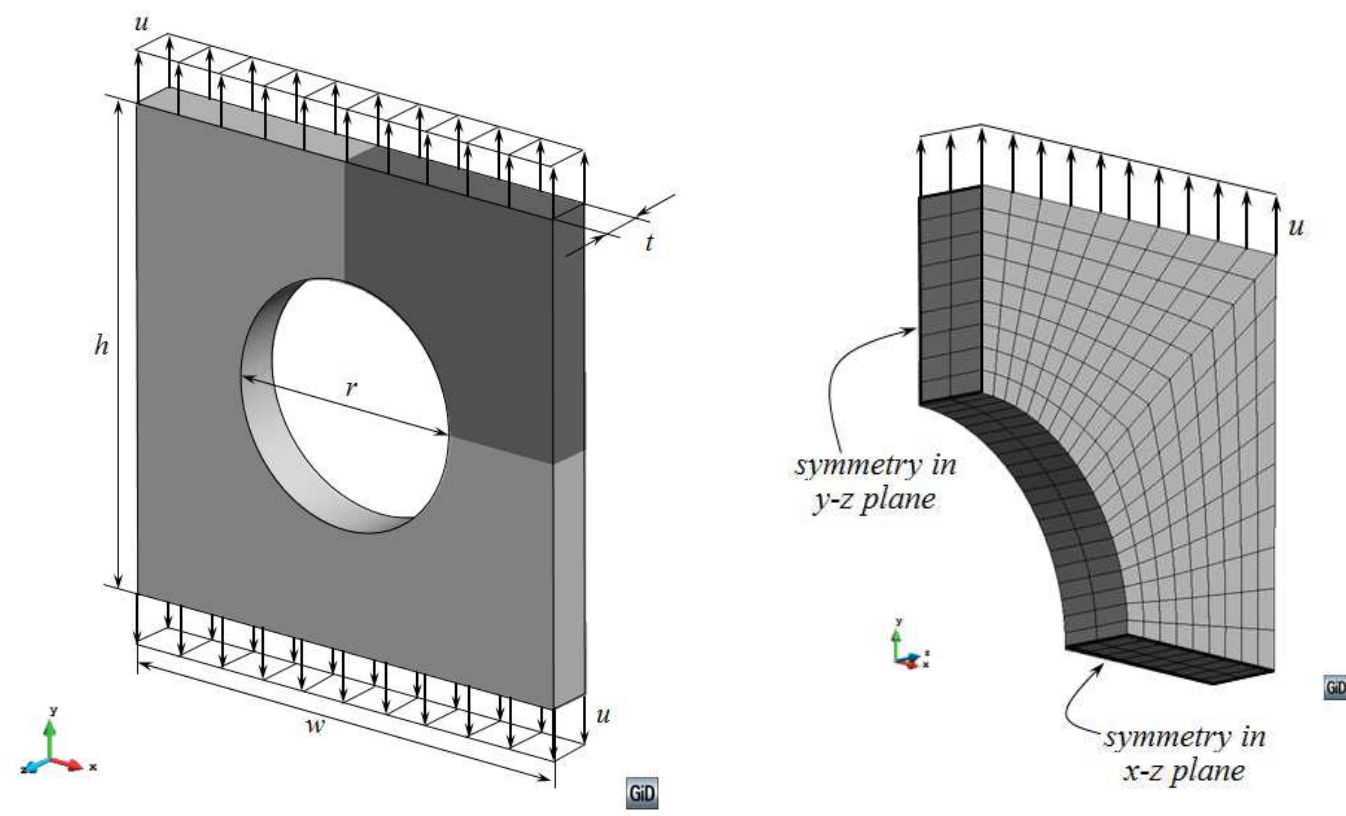

Figure 7. Geometry ( $r=100 \mathrm{~mm}, h=w=200 \mathrm{~mm}, t=20 \mathrm{~mm}$ ) and loading of the membrane with a hole as described in [44] (left); and mesh and boundary conditions imposed on the quarter of the membrane which has been discretized (right).

defined in Figure 4, except for the initial damage threshold and the fracture energy which are set to $\mathrm{Td}=34.7 \mathrm{~Pa}^{1 / 2}$ and $\mathrm{Gf}=1200 \mathrm{kN} / \mathrm{m}$, respectively.

The mechanical response of the membrane with neo-Hookean-based damage formulation is illustrated in Figure 8 (top left) by means of the vertical reaction vs. stretch curve. The vertical reaction plotted is the total resultant reaction force in the $y$-direction of the quarter of the specimen. It can be observed how the initial response of the curve follows the undamaged (hyperelastic) load path, depicted as a gray dotted line in the figure, up to approximately a displacement value of $u=15 \mathrm{~mm}$. This point corresponds to the initiation of damage in the specimen, whose progression results in a considerable reduction of the overall structural stiffness. Figure 8 (bottom) shows the distribution of the damage variable, $D$, in the specimen for the displacement values $u=20,28$ and $47 \mathrm{~mm}$.

Damage initiates in the bottom corner of the quarter hole and progresses horizontally in the outward direction, localizing for the lower band of elements. This localization allows verifying that energy dissipation is being computed correctly following the calculations described in the Appendix. As these elements where damage has localized are increasingly damaged, loosing, thus, the stiffness of their deviatoric part, they become largely deformed. However, the quasi-incompressible character of the hybrid elements requires that the adjacent band of elements deform to accommodate the narrowing of the highly damaged elements in the lower band. This, in turn, generates higher deviatoric stresses in these adjacent row of elements, which result in damage initiation.

The convergence curves for each load increment, plotted in Figure 8 (top right), show adequate convergence of the solution. Note that a tolerance of $10^{-7}$ has been used. 

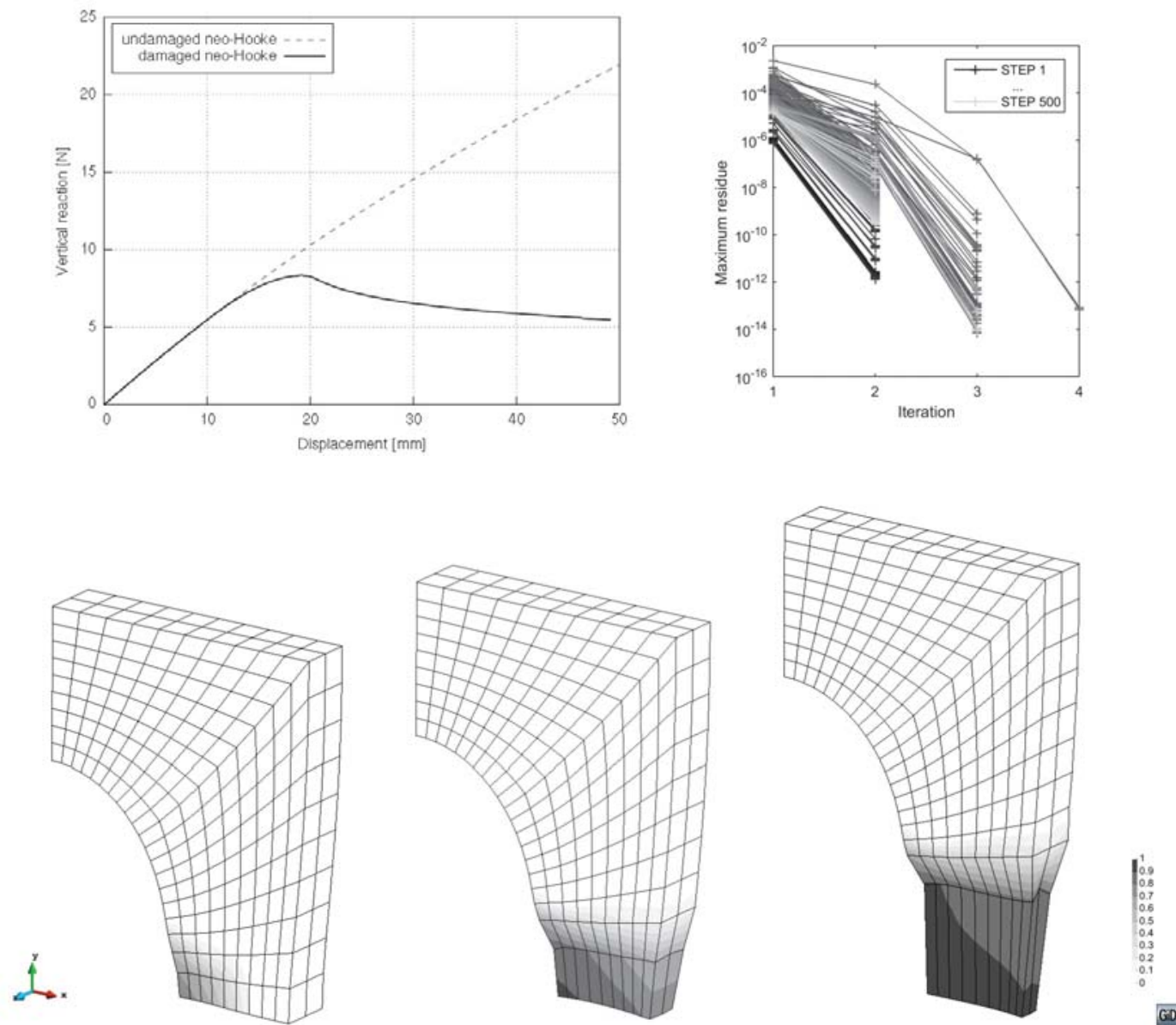

Figure 8. Neo-Hookean-based damage model with an initial damage threshold $\mathrm{Td}=57.7 \mathrm{~Pa}^{1 / 2}$ and fracture energy $\mathrm{Gf}=600 \mathrm{kN} / \mathrm{m}$. Vertical reaction vs. stretch response (top left) and convergence curves of each load step (top right). Damage distribution, $D$, of this specimen corresponding to the imposed displacement values, $u$, of $20 \mathrm{~mm}$ (bottom left), $28 \mathrm{~mm}$ (bottom center) and $47 \mathrm{~mm}$ (bottom right). Real deformation $(\times 1)$ is plotted.

The vertical reaction of the membrane with Ogden-based damage formulation is plotted vs. the stretch in Figure 9 (top left). In this case, the value of the vertical reaction continues increasing once damage initiates in the structure at approximately $u=20 \mathrm{~mm}$, albeit at a considerably slower rate than the expected load path of the corresponding undamaged (hyperelastic) model, depicted as a gray dotted line. This effect is analogous to the one observed in the stress vs. stretch curves of Figure 6, where the stiffness increase of the undamaged model is much higher than the decrease induced by damage softening on the deviatoric part of the stress. However, damage softening is still occurring since the damaged response exhibits lower stiffness than the original undamaged hyperelastic model. Thus, the damage formulation proposed is capable of representing a wide range of damage softening behaviors including both positive and negative slopes in the load-displacement or stress-stretch response.

As in the neo-Hookean-based model, damage also initiates in the bottom corner of the quarter hole but now progresses differently, as seen in Figure 9 (bottom). In this case, damage does not localize in a band of elements, instead, it propagates vertically at first and, then, outward, resulting 

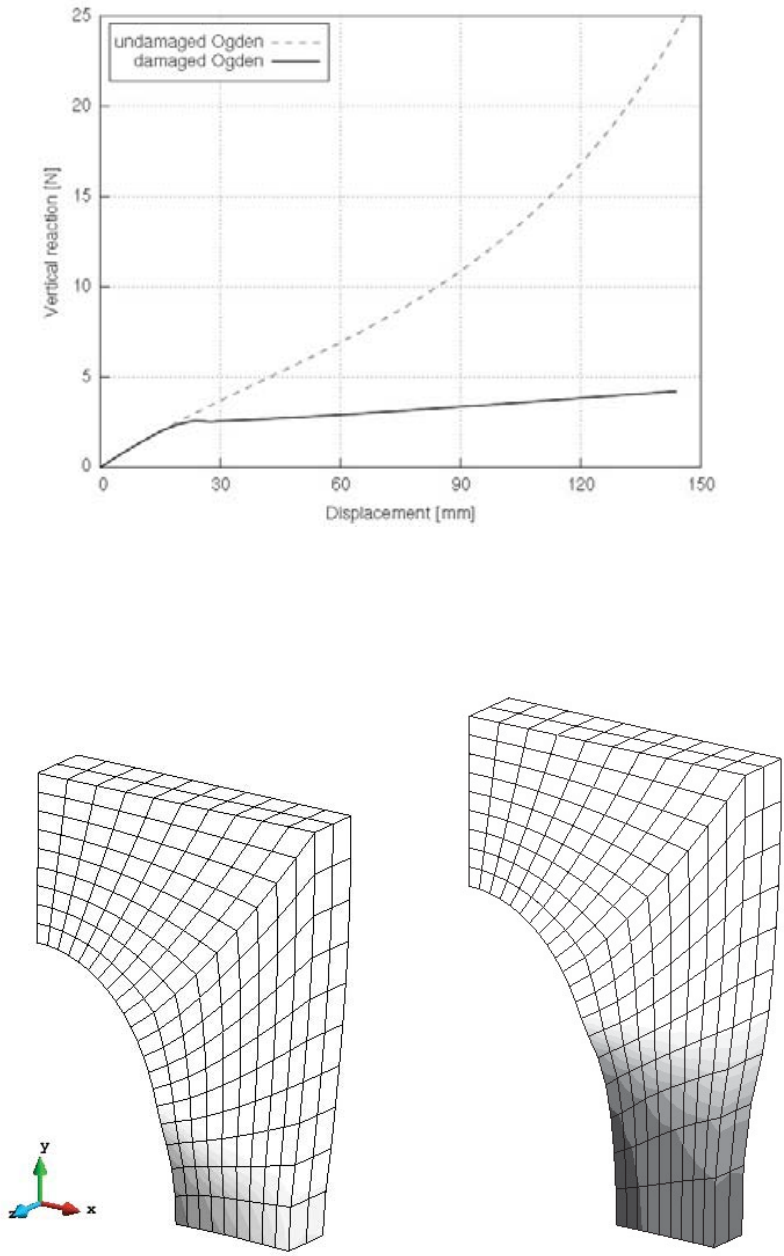
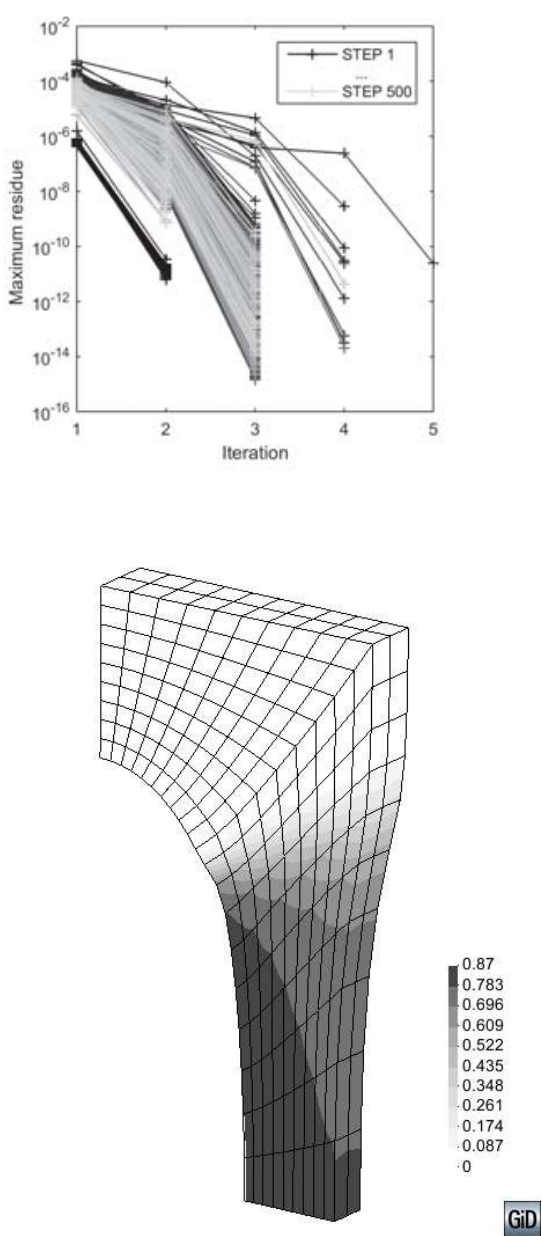

Figure 9. Ogden-based damage model with an initial damage threshold $\mathrm{Td}=34.7^{1 / 2}$ and fracture energy $\mathrm{Gf}=1200 \mathrm{kN} / \mathrm{m}$. Vertical reaction vs. stretch response (top left) and convergence curves of each load step (top right). Damage distribution, $D$, of this specimen corresponding to the imposed displacement values, $u$, of $27 \mathrm{~mm}$ (bottom left), $44 \mathrm{~mm}$ (bottom center) and $76 \mathrm{~mm}$ (bottom right). Real deformation $(\times 1)$ is plotted.

in a much larger zone of the structure affected by damage. Note that the displacements imposed in this model are three times as large as those imposed in the neo-Hookean-based one, therefore, stress induced by them will also be larger and probably increases faster than the damage propagation rate that would be required for localization in the lower band of elements.

The convergence curves for each load increment, plotted in Figure 9 (top right), show adequate convergence of the solution. Note that a tolerance of $10^{-7}$ has been used.

\subsection{Damage in fibered soft biological tissue}

The damage formulation proposed in this paper has been developed with the aim of representing the behavior of fibered soft biological tissues by means of mixing theory. This theory provides the behavior of a composite material as the composition of the individual components according to their particular morphology and mechanical properties. For a more detailed description of the theoretical basis of mixing theory see, for example, reference [40] or [41]. 
Since mixing theory is, in fact, a constitutive equation manager, it allows for disparate material behaviors of its individual components, each representable by a completely different constitutive equation. To the best knowledge of the authors, the constitutive models used up to date to represent damage in fibered soft tissues integrate the contribution of the fibers and matrix at strain-energy function level [31, 32, 33, 34, 43]. The mixing theory approach manages the contribution of fiber and matrix at stress level. This allows for more flexibility in composing the overall behavior of the tissue since the fiber and matrix can be easily modeled with different constitutive equations if required. In addition, the simple constituent models used have a solid and established thermodynamical basis, which allows for better tracing of the individual component's thermo-mechanical behavior.

In this context, the proposed damage model can be used to represent damage in either fiber or matrix, or in both. Then, the contribution of each component to the composite tissue is determined by their volumetric participation (v). As an example, the experimental data obtained by Martins et al. [27] is used to illustrate how the manifestly different behaviors of fiber and matrix can be represented by means of the damage model proposed, particularized for Ogden hyperelasticity, and in the framework of mixing theory.

The work by Martins et al. provides experimental stress-stretch curves obtained from an uniaxial tensile test of a rectus sheath sample in the longitudinal and transversal directions. Using Matlab's Curve Fitting Toolbox [42], an initial estimate of the material parameters of fiber and matrix were obtained, which were then adjusted in the numerical reproduction of the sample to better fit the experimental curve. The material parameters used are given in Figure 10, together with the stressstretch curve numerically obtained using mixing theory and the Ogden-based damage formulation implemented in the in-house code PLCd. Due to lack of information, the fiber contribution to the composite was estimated as $20 \%$ of the composite, based on information available in literature. A different proportion of fiber and matrix in the composite would obviously lead to a completely different stress response of the fiber in order to fit the composite response with the experimental data.

\section{CONCLUSIONS}

A new generalized damage model for quasi-incompressible hyperelasticity in a total Lagrangian finite strain framework has been presented and discussed. The damage model is based on the decoupled volumetric-isochoric definition of quasi-incompressible hyperelastic formulations. These require the use of hybrid elements, in which an additional variable, pressure, must be computed from the equations of motion in addition to the displacements.

A Kachanov-like reduction factor is applied on the deviatoric part of the hyperelastic constitutive model. Linear and exponential softening have been defined as damage evolution laws, both translated from an infinitesimal strain context to the present finite strain framework. Other softening laws could be considered to model particular materials. However, the evolution laws presented here have the advantage of a straightforward formulation and being easily adaptable to model different material behaviors since they are defined only by the material properties initial damage threshold, $\tau_{0}^{d}$, and maximum dissipated fracture energy per unit volume, $g_{f}^{d}$. Also, the popular Simo and Ju damage criterion has been used, but any other energy-based criterion could be easily introduced. 


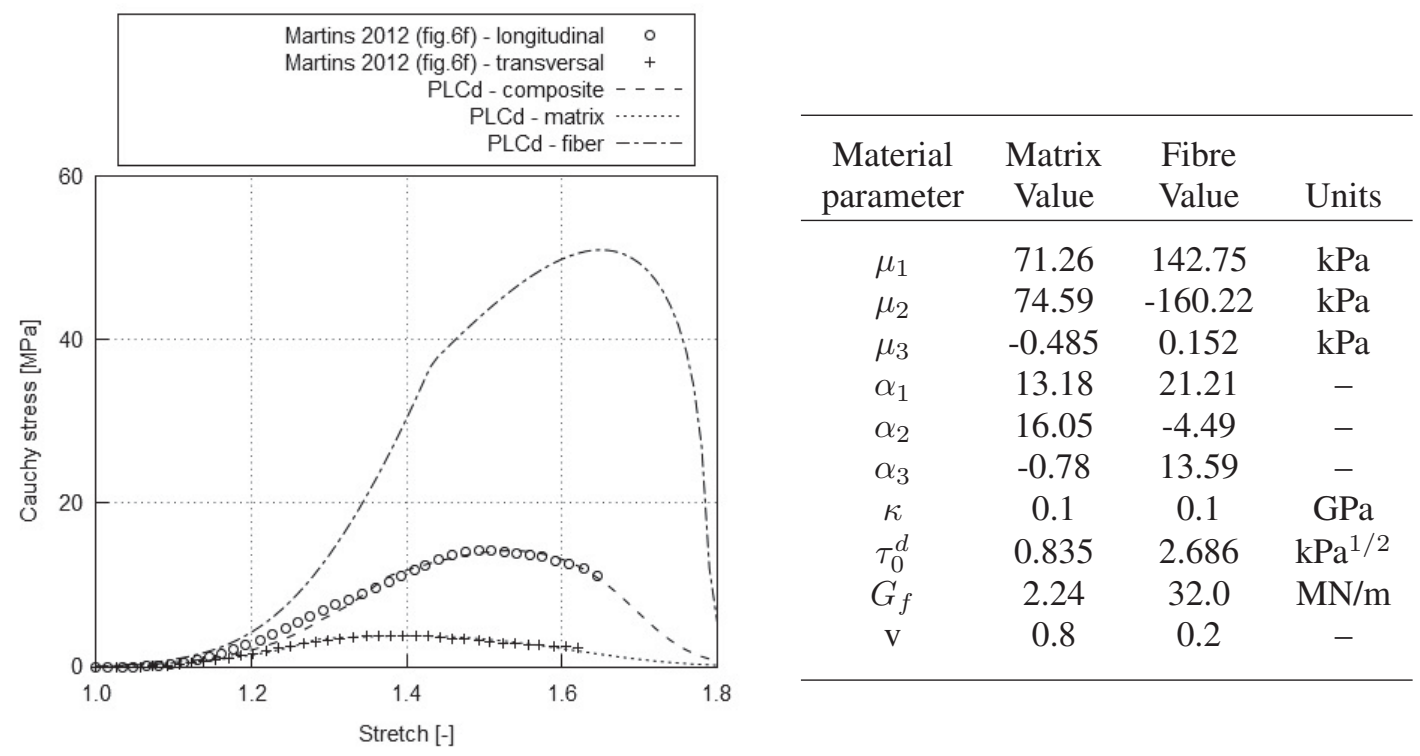

Figure 10. Cauchy stress vs. stretch of the composite and its individual components modeled with the Ogdenbased damage formulation and mixing theory to reproduce the experimental data by Martins et al. [27] (left) and the material parameters used (right).

The generalized damage model has been particularized for two types of hyperelastic formulation, neo-Hooke and Ogden hyperelasticity, and implemented in the in-house finite element code PLCd. Examples have been presented in order to illustrate the main characteristics of the proposed damage model. The damage variable used has been shown to be accumulative and discontinuous, as imposed by the Kuhn-Tucker conditions.

The damage formulation particularized for Ogden hyperelasticity has been used to fit experimental data of fibered soft biological tissue [27] by means of mixing theory. This theory describes the behavior of a composite material as the composition between the individual components. In this case, both fiber and matrix have been modeled with the aforementioned damage formulation. However, the constituents in mixing theory can be modeled with any desired constitutive equation, which allows for a wide range of possible material behaviors and, thus, a large variety of overall composite responses. Although the damage model presented in this paper is isotropic, further work includes introducing anisotropy at composite level through the mixing theory formulation.

The damage softening approach presented is robust and versatile. It can be easily adapted to any desired hyperelastic formulation as long as it is defined with split volumetric and deviatoric parts. In addition, it is able to reproduce a wide range of softening behaviors, as made clear in the numerical examples. However, one must bear in mind that the non-linear nature of the undamaged formulations influences greatly the final softening behavior of the damage model. Unlike in the infinitesimal strain context, the linearity or exponentiality of the damage evolution law does not directly dictate the shape of the softening curve in the present model.

Furthermore, the use of quasi-incompressible elements makes it difficult for damage to localize in a band of elements as is common in infinitesimal strain damage models. Damaged elements loose part of their stiffness, stretching in the loading direction. Due to incompressibility, the narrowing 
of these elements require the adjacent elements to deform accordingly, inducing higher deviatoric stresses in them which, in turn, results in damage. Note, however, that Q1P0 elements have been used, which require fine meshing due to the lack of compliance with the inf-sup condition. Improving the $u / p$ elements will predictably result in better results, especially in terms of damage localization and evolution in complex geometries. In any case, the fact that damage is applied only on the deviatoric part of the model means that, for a completely damaged structure, there will always remain a volumetric quasi-incompressible undamaged part.

\section{A. CALCULATION OF THE DISSIPATION}

The total dissipation value of the structure, $W_{f}$, is numerically obtained by means of expression (5) as follows

$$
W_{f}=\int_{V} \int_{t_{0}}^{t_{\infty}} \Xi d t d V
$$

When damage localizes in a band of elements, this can be compared to an estimate of the same value calculated in terms of the fracture energy, taking into account (11), and the final volume of the elements in the damaged band as

$$
W_{f}=\int_{V} g_{f}^{d} d V=g_{f}^{d} V_{f}
$$

where the maximum dissipated fracture energy per unit volume, $g_{f}^{d}$, is related to the material property $G_{f}$, which is the maximum dissipated fracture energy per unit area, through the element's characteristic length in the reference configuration, $L_{0}: g_{f}^{d}=G_{f} / L_{0}$. The final volume can be computed as $V_{f}=A_{f} l_{f}$, where $A_{f}$ is the final cross-section area of the band of elements where damage has localized and $l_{f}$ is the final length of these elements in the direction perpendicular to $A_{f}$. Finally, defining a final damage stretch as $\lambda_{f}=l_{f} / L_{0}$, the expression for the total dissipation results in

$$
W_{f}=\lambda_{f} A_{f} G_{f}
$$

\section{ACKNOWLEDGEMENT}

This work was partially supported by the European Research Council under the Advanced Grant: ERC2012-AdG 320815 COMP-DES-MAT “Advanced tools for computational design of engineering materials". E. Comellas gratefully acknowledges the support of the Departament d'Universitats, Recerca i Societat de la Informació (DURSI) of the Generalitat de Catalunya through an FI-AGAUR scholarship.

\section{REFERENCES}

1. Kachanov LM. Rupture time under creep conditions. International Journal of Fracture 1999; 97(1):11-18. DOI: 10.1023/A:1018671022008.

2. Kachanov LM. Introduction to continuum damage mechanics (Vol. 10). Dordrecht: Springer Netherlands, 1986. DOI:10.1007/978-94-017-1957-5. 
3. Simo JC, Ju JW. Strain- and stress-based continuum damage models - Parts I and II. International Journal of Solids and Structures 1987; 23(7):821-869. DOI:10.1016/0020-7683(87)90083-7 and 10.1016/0895-7177(89)90118-0.

4. Chaboche JL. Continuum damage mechanics: Part I - General concepts. Journal of Applied Mechanics 1988; 55(1):59-64. DOI: 10.1115/1.3173661.

5. Lemaitre J. A Course on Damage Mechanics. Berlin: Springer. 1996. DOI:10.1007/978-3-642-18255-6.

6. Murakami S. Notion of continuum damage mechanics and its application to anisotropic creep damage theory, Journal of Engineering Materials and Technology 1983; 105(2):99-105. DOI:10.1115/1.3225633.

7. Ju JW, Isotropic and anisotropic damage variables in continuum damage mechanics, Journal of Engineering Mechanics 1990; 116(12):2764-2770. DOI:10.1061/(ASCE)0733-9399.

8. Lemaitre J. Coupled elasto-plasticity and damage constitutive equations Computer Methods in Applied Mechanics and Engineering 1985; Comput. Methods Appl. Mech. Engrg. 51(1-3):31-49. DOI:10.1016/0045-7825(85)90026$\mathrm{X}$.

9. Ju JW. On energy-based coupled elastoplastic damage theories: Constitutive modeling and computational aspects. International Journal of Solids and Structures 1989; 25(7):803-833. DOI:10.1016/0020-7683(89)90015-2.

10. Oller S, Oliver J, Lubliner J, Oñate E. Un modelo de daño plastico para materiales friccionales. Parts I and II. Revista Internacional de Métodos Numéricos para Cálculo y Diseño en Ingeniería 1988; 4(4):397-431.

11. Faria R, Oliver J, Cervera M. A strain-based plastic viscous-damage model for massive concrete structures. International Journal of Solids and Structures 1998; 35(14):1533-1558. DOI:10.1016/S0020-7683(97)00119-4.

12. Lubliner J, Oliver J, Oller S, Oñate E. A plastic-damage model for concrete. Parts I and II. International Journal of Solids and Structures 1989; 25(3):299-326. DOI:10.1016/0020-7683(89)90050-4.

13. Ortiz M. A constitutive theory for the inelastic behavior of concrete. Mechanics of Materials 1985; 4(1) 67-93. DOI:10.1016/0167-6636(85)90007-9.

14. Oller S, Oñate E, Miquel J, Botello S. A plastic damage constitutive model for composite materials. International Journal of Solids and Structures 1996; 33(17):2501-2518. DOI:10.1016/0020-7683(95)00161-1.

15. Hokanson J, Yazdani S. A constitutive model of the artery with damage. Mechanics Research Communications 1997; 24(2):151-159. DOI: 10.1016/S0093-6413(97)00007-4.

16. Simo JC. On a fully three-dimensional finite-strain viscoelastic damage model: Formulation and computational aspects. Computer Methods in Applied Mechanics and Engineering 1987; 60(2):153-173. DOI: 10.1016/00457825(87)90107-1.

17. Miehe C. Discontinuous and continuous damage evolution in Ogden-type large-strain elastic materials. European journal of mechanics. A. Solids 1995; 14(5):697-720.

18. de Souza Neto EA, Perić D, Owen DRJ. Continuum modelling and numerical simulation of material damage at finite strains. Archives of Computational Methods in Engineering 1998; 5(4):311-384. DOI: 10.1007/BF02905910.

19. Diani J, Fayolle B, Gilormini P. A review on the Mullins effect. European Polymer Journal 2009; 45(3):601-612. DOI: 10.1016/j.eurpolymj.2008.11.017.

20. Peña E, Peña JA, Doblaré M. On the Mullins effect and hysteresis of fibered biological materials: A comparison between continuous and discontinuous damage models. International Journal of Solids and Structures 2009; 46(78):1727-1735. DOI: 10.1016/j.ijsolstr.2008.12.015.

21. Balzani D, Brinkhues S, Holzapfel GA. Constitutive framework for the modeling of damage in collagenous soft tissues with application to arterial walls. Computer Methods in Applied Mechanics and Engineering 2012; 213216(0):139-151. DOI: 10.1016/j.cma.2011.11.015.

22. Oller S. Nonlinear dynamics of structures Springer. 2014.

23. Truesdell C, Toupin R. The Classical Field Theories. In S. Flgge (Ed.), Principles of Classical Mechanics and Field Theory / Prinzipien der Klassischen Mechanik und Feldtheorie 1960; 226858. Berlin:Springer. DOI:10.1007/9783-642-45943-6-2.

24. Rastellini F, Oller S, Salomón, Onate E. Composite material non-linear modelling for long fibre-reinforced laminates. Continuum basis, computational aspects and validations. Computers \& Structures 2008; 86(9):879-896.

25. PLCd Manual. Non-linear thermo-mechanic finite element code oriented to PhD student education. Code developed at CIMNE; 1991-to present. URL: http://https//web.cimne.upc.edu/users/plcd/

26. Schenk O, Gärtner K. Solving unsymmetric sparse systems of linear equations with PARDISO. Future Generation Computer Systems 2004; 20(3):475-487.

27. Martins P, Peña E, Jorge RMN, Santos A, Santos L, Mascarenhas T, Calvo B. Mechanical characterization and constitutive modelling of the damage process in rectus sheath. Journal of the Mechanical Behavior of Biomedical Materials 2012; 8:111-122. DOI:10.1016/j.jmbbm.2011.12.005.

28. Simo JC, Taylor RL. Quasi-incompressible finite elasticity in principal stretches. Continuum basis and numerical algorithms. Computer Methods in Applied Mechanics and Engineering 1991; 85(3):273-310. DOI: 10.1016/00457825(91)90100-K. 
29. Holzapfel GA. Nonlinear solid mechanics: A continuum approach for engineering. England: John Wiley \& Sons, 2001.

30. Oliver J, Cervera M, Oller S and Lubliner J. Isotropic damage models and smeared crack analysis of concrete, In Proc. SCI-C Computer Aided Analysis and Design of Concrete Structures 1990; 945958.

31. Natali AN, Pavan PG, Carniel EL, Lucisano ME, Taglialavoro G. Anisotropic elasto-damage constitutive model for the biomechanical analysis of tendons. Medical Engineering \& Physics 2005; 27(3):20914. DOI: 10.1016/j.medengphy.2004.10.011.

32. Rodríguez JF, Cacho F, Bea JA, Doblaré M. A stochastic-structurally based three dimensional finite-strain damage model for fibrous soft tissue. Journal of the Mechanics and Physics of Solids 2006; 54(4):864886. DOI: 10.1016/j.jmps.2005.10.005.

33. Calvo B, Peña E, Martínez MA, Doblaré M. An uncoupled directional damage model for fibred biological soft tissues. Formulation and computational aspects. International Journal for Numerical Methods in Engineering 2007; 69(10):2036-2057. DOI: 10.1002/nme.1825.

34. Peña E, Calvo B, Martínez MA; Doblaré M. On finite-strain damage of viscoelastic-fibred materials. Application to soft biological tissues, International Journal for Numerical Methods in Engineering 2008; 74(7):1198-121. DOI:10.1002/nme.2212.

35. Sussman T, Bathe K-J. A finite element formulation for nonlinear incompressible elastic and inelastic analysis. Computers \& Structures 1987; 26(1/2):357-409. DOI: 10.1016/0045-7949(87)90265-3.

36. Crisfield MA. Non-linear finite element analysis of solids and structures. England: John Wiley \& Sons, 1991.

37. Bathe K-J. Finite element procedures. New Jersey: Prentice-Hall, 1996.

38. Bonet J, Wood RD. Nonlinear continuum mechanics for finite element analysis. Cambridge: Cambridge University Press, 2008.

39. Salomón O, Oller S, Barbat A. Un tratamiento de material tipo goma, mediante la condensación de la variable de presión. Mecánica Computacional 2009; Vol XVII. Num 6. Solid Mechanics (C) 361-372.

40. Car E, Oller S, Oñate E. An anisotropic elastoplastic constitutive model for large strain analysis of fiber reinforced composite materials. Computer Methods in Applied Mechanics and Engineering 2000; 185(2-4):245277. DOI:10.1016/S0045-7825(99)00262-5.

41. Oller S. Numerical simulation of mechanical behavior of composite materials. Springer. 2014.

42. MATLAB and Curve Fitting Toolbox Release 2014b, The MathWorks Inc., Massachusetts, United States.

43. Peña E. Computational aspects of the numerical modelling of softening, damage and permanent set in soft biological tissues. Computers \& Structures 2014; 130:57-72.

44. Waffenschmidt T, Poliandara C, Menzel A and Blanco S. A gradient-enhanced large-deformation continuum damage model for fibre-reinforced materials. Computer Methods in Applied Mechanics and Engineering 2014; 268:801-842. 